\title{
Low distortion fast light in an optical fiber using stimulated Brillouin scattering
}

\author{
Luc Thévenaz, Sanghoon Chin and Miguel Gonzalez-Herraez* \\ Ecole Polytechnique Fédérale de Lausanne, STI-NAM Station 11, CH-1015 Lausanne, Switzerland \\ * Department of Electronics, University of Alcalá de Henares, E-28805 Madrid, Spain \\ luc.thevenaz@epfl.ch, sanghoon.chin@epfl.ch, miguelg@depeca.uah.es
}

\begin{abstract}
We demonstrate experimentally a novel approach for fast light generation based on a wideband compound spectral resonance using stimulated Brillouin scattering. The pulses experience fast light with extremely reduced distortion and small amplitude change.

(C)2007 Optical Society of America

OCIS codes: (060.4370) Nonlinear optics, fibers; (290.5900) Scattering, stimulated Brillouin; (350.5500) Propagation
\end{abstract}

\section{Introduction}

Recently, slow \& fast light has received tremendous interest from optical communication community because it opens up interesting opportunities to achieve all-optical delay lines and to provide a timing tool for photonics signal processing [1]. Fast light experiments are particularly fascinating since they make possible very extreme propagation condition such as superluminal and negative velocities. In general, these experiments require the presence of a large anomalous dispersion in the medium near the signal frequency. Sharp atomic absorptions and electromagnetically induced absorption have provided means to create a large anomalous dispersion. In optical fibers, the narrowband absorption of stimulated Brillouin scattering (SBS) has been used to produce these conditions [2]. All these methods to generate fast light, however, have the common drawback of making the signal pulse propagate in a spectral region of high absorption. To overcome this impairment, we proposed a solution that makes use of two close SBS resonances generated by a two-tone pump. In between the resonances, a spectral region of anomalous dispersion appears, in which gain-assisted fast light was experimentally realized [3]. However, the signal distortion in this experiment was still far from acceptable for real applications and the underlying scientific challenge to produce pulse advancement and fast light with negligible distortion remains intact.

In this paper, we propose an improved method to generate fast light with low distortion and small power variation. We use a bichromatic pump source as in the previous work to generate by the SBS process two widely separated gain resonances In addition the bandwidths of the resonances are controlled by simply broadening the pump spectrum in order to maximize the delay/distortion characteristics in the fibers [4]. 


\section{JTuA8.pdf}

\section{Principle}

The SBS in single mode optical fibers is a nonlinear interaction between two counter-propagating waves, a strong pump wave and a weak probe wave. Once a particular phase matching condition is fulfilled, an acoustic wave is generated by electrostriction and scatters photons from the pump to the probe, leading to a narrowband resonance near the probe frequency. Thus simply using a double-frequency pump allows us to achieve two separate SBS gain bands with identical spectra and an anomalous dispersion in turn appears in the middle of the gain doublet. Therefore, pulse advancement or fast light can be realized when the signal pulse is spectrally set in the valley between the two peaks. Moreover, the pump laser is directly modulated by a noise generator to broaden its spectrum [4] and to modify the effective spectral profile of the Brillouin gain doublet. This way the gain spectral shape is precisely chosen so as to optimize the delay/distortion characteristics of the medium that gives rise to maximum pulse advancement with low distortion and small power variation.

\section{Experiments and Results}

A conventional single mode optical fiber with a length of $2 \mathrm{~km}$ is used as the SBS gain medium. We measured the Brillouin characteristics of this fiber, resulting in a Brillouin shift of $10.8 \mathrm{GHz}$ and an SBS gain bandwidth of 25 MHz. A commercial DFB laser emitting at $1532 \mathrm{~nm}$ is used to generate the double-frequency pump beam. The laser light is modulated by means of a Mach-Zehnder EOM driven with a $100 \mathrm{MHz}$ tone. The DC bias voltage on the modulator is accurately adjusted, so that a full extinction of the carrier is achieved. As a consequence, the optical spectrum at the modulator output contains only two sidebands that create two close SBS gain resonances with identical spectra, showing a separation between central frequencies of $200 \mathrm{MHz}$ with perfect inherent stability. Moreover, we applied a direct modulation to the pump laser in order to broaden its spectrum to achieve an optimized spectral shape in order to maximize the pulse advancement with low distortion. The laser current is directly modulated by use of a noise generator and its spectral width is slightly broadened to $44 \mathrm{MHz}$. This way we obtained a wideband quasi parabolic spectral distribution of FWHM $160 \mathrm{MHz}$ across the two peaks. The probe pulse train is produced from a distinct DFB laser operating at the same wavelength of $1532 \mathrm{~nm}$. The temperature and bias current of the probe laser are precisely adjusted to ensure propagation in the dip between the two gain resonances. The laser is fast-optically gated using another EOM to generate a pulse train. This pulse in turn enters into the fiber and counter-propagates with respect to the pump.

A gain doublet is generated after propagating through the $2 \mathrm{~km}$ optical fiber as shown in Figure.1-(a). It must be pointed out that the Brillouin spectrum in the case of modulation is much smoother than in the unmodulated case. 


\section{JTuA8.pdf}

The overlapping of the two gain distributions obtained using the modulated pump gives rise to a perfect linear transition in the effective refractive index that results in fast light propagation with minimal distortion. Positioning spectrally the signal in the dip between the two gain peaks offers the further advantage to give a minimal amplification while fully maintaining the delaying effect. The FWHM pulse width used in this experiment is 6.6 ns and Figure.1-(b) shows the non-normalized time waveforms of the pulse after passing through the fiber. The pulses are clearly advanced with respect to the pump power and show a strongly reduced distortion.
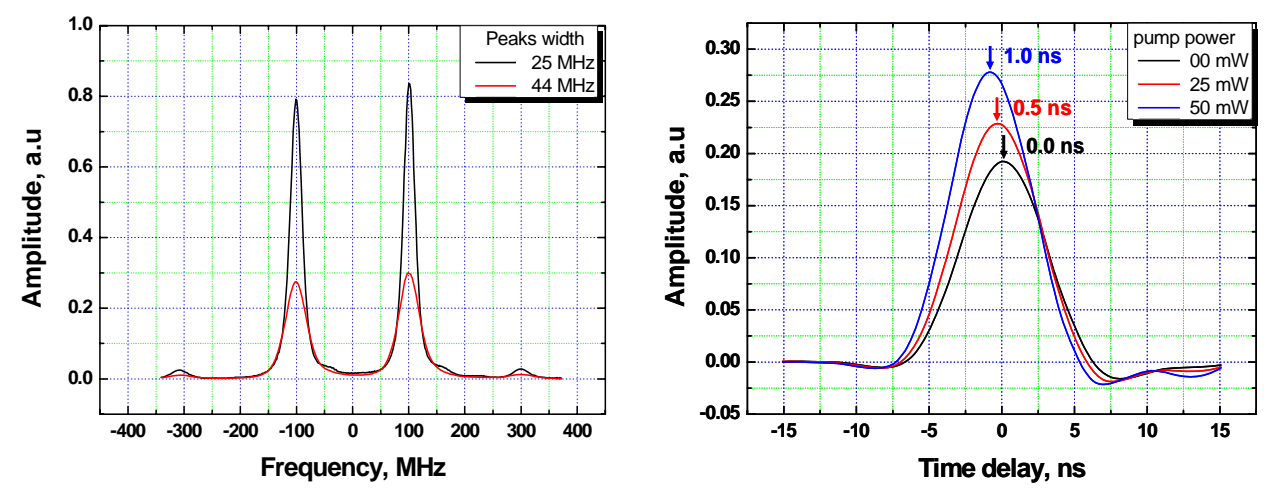

Fig.1. (a) The SBS doublet generated by the spectrally broadened double frequency pump. (b) The typical traces of the probe pulses after propagating through the fiber for different pump powers, showing clear pulse advancement with a strongly reduced distortion.

\section{Conclusions}

In this paper, we have demonstrated experimentally that a signal pulse can be advanced with a strongly reduced distortion and small amplitude change through optical fibers. The scheme is very flexible and can match any signal bandwidth up to several GHz by simply increasing the frequency doublet separation and the spectral broadening. It makes possible a fine optimization of the distortion and the advancement by properly setting the amount of broadening with respect to the doublet separation. The scheme can also be applied for slow light generation by propagating in the SBS loss spectral region.

\section{References}

1. R. W. Boyd and D.J. Gauthier, “'Slow' and 'Fast' light,” Ch. 6 in Progress in Optics 43, E. Wolf, Ed. (Elsevier, Amsterdam, 2002), 497-530.

2. K. Y. Song, M. Gonzalez Herráez and L. Thévenaz, Opt. Express 13, 82-88 (2005).

3. K. Y. Song, M. Gonzalez Herráez and L. Thévenaz, Opt. Express 13, 9758-9765 (2005).

4. M. Gonzalez Herráez, K. Y. Song and L. Thévenaz, Opt. Express 14, 1395-1400 (2006). 